\title{
Seleção de isolados de Beauveria bassiana patogênicos ao bicudo-do-algodoeiro(1)
}

\author{
Carlos Alberto Domingues da Silva(2)
}

\begin{abstract}
Resumo - Objetivou-se selecionar isolados de Beauveria bassiana (Balsamo) Vüillemin provenientes de diferentes hospedeiros e regiões geográficas, patogênicos ao Anthonomus grandis, o bicudo-doalgodoeiro. Foram analisados 12 isolados em condições de laboratório. Os isolados obtidos originalmente de $A$. grandis foram pouco virulentos a essa praga. A mortalidade do bicudo teve início no segundo dia após a inoculação das suspensões fúngicas, variando de $15 \%$ a $83 \%$, com $\mathrm{TL}_{50}$ entre 5,30 a 11,06 dias. Os isolados apresentaram variabilidade quanto à germinação dos conídios em meio de cultura artificial, e esta não se correlacionou com a patogenicidade. O isolado CG138 de B. bassiana destacou-se como um dos mais virulentos ao bicudo-do-algodoeiro.
\end{abstract}

Termos para indexação: Anthonomus grandis, algodão, patógenos, patogenicidade, pragas de plantas.

\section{Selection of isolates of Beauveria bassiana pathogenic to cotton boll weevil}

\begin{abstract}
The objective of this work was to screen pathogenic isolates of Beauveria bassiana (Balsamo) Vüillemin from different hosts and geographic regions, pathogenic to the cotton boll weevil. Twelve isolates were evaluated. Isolates originally obtained from boll weevil showed low pathogenicity against this host. Insect mortality began on the second day after inoculation by fungal suspension, ranging from $15 \%$ to $83 \%$, with a $\mathrm{TL}_{50}$ of 5.30 to 11.06 days. Variability among isolates concerning conidial germination in artificial culture medium was verified, and $\mathrm{TG}_{50}$ did not correlate with pathogenicity. One of the most virulent isolates of B. bassiana against cotton boll weevil is CG138.
\end{abstract}

Index terms: Anthonomus grandis, cotton, pathogens, pathogenicity, pests of plants.

\section{Introdução}

Existem várias referências na literatura demonstrando a importância dos microrganismos entomopatogênicos no controle de diversas pragas agrícolas. Dentre esses, os fungos merecem destaque por serem os microorganismos mais freqüentemente encontrados atacando insetos.

Atualmente, cerca de 25 espécies são reconhecidas como importantes fatores bióticos no controle de insetos economicamente preocupantes para as áreas da agricultura e medicina (McCoy \& Tigano-Milani, 1992). Diferentemente das bactérias, protozoários e

\footnotetext{
(1) Aceito para publicação em 29 de novembro de 2000. Pesquisa financiada pelo Common Fund for Commodities, International Cotton Advisory Committee.

(2) Embrapa-Centro Nacional de Pesquisa de Algodão, Caixa Postal 174, CEP 58107-720 Campina Grande, PB. E-mail: carlos@cnpa.embrapa.br
}

vírus, os fungos podem infectar os insetos não somente pelo intestino, mas também pelos espiráculos e particularmente pela superfície do tegumento. Esta propriedade conduz à possibilidade teórica da infecção de insetos independentemente de sua atividade alimentar (Hajek \& Leger, 1994).

Nos Estados Unidos, diversas formulações do fungo entomopatogênico Beauveria bassiana (Bals.) Vuill. têm sido testadas com sucesso no controle do bicudo-do-algodoeiro, Anthonomus grandis (Coleoptera: Curculionidae), tanto em laboratório (Wright \& Chandler, 1991) quanto no campo (Frank \& Slosser, 1990; Wright \& Chandler, 1992; Wright, 1993). Wright (1993) testou a eficiência de um micoinseticida à base de $B$. bassiana no controle de adultos do bicudo nos anos de 1991 e 1992. De acordo com esse autor, o controle do bicudo no tratamento pulverizado com micoinseticida não diferiu do tratamento pulverizado com inseticida químico no ano de 1991, porém é um pouco menos eficiente no ano seguinte, em relação ao inseticida químico. 
No Brasil, o B. bassiana tem-se destacado como agente de controle biológico com potencial para ser empregado no controle de populações de adultos do bicudo-do-algodoeiro (Coutinho \& Cavalcanti, 1988; Coutinho \& Oliveira, 1991). Apesar disso, seu uso na cultura do algodão para o controle do bicudo tem sido limitado, em parte, por resultados inconsistentes obtidos nas aplicações de campo. É possível que os isolados utilizados nessas pesquisas não se tenham adaptado às condições climáticas encontradas nos campos onde o algodão é cultivado. Assim, em um programa de melhoramento genético de microorganismos usados para o controle biológico, deve-se empregar, em primeiro lugar, processos mais simples e naturais, e depois de esgotá-los, partir para os mais sofisticados (Melo \& Azevedo, 1998). Considerando que o ambiente determina o biótipo dos patógenos, é provável que a virulência e agressividade do fungo varie de acordo com a espécie de hospedeiro e a região geográfica considerada.

Este trabalho teve como objetivo selecionar isolados de B. bassiana provenientes de diferentes hospedeiros e regiões geográficas, que sejam virulentos ao bicudo-do-algodoeiro.

\section{Material e Métodos}

O experimento foi realizado no Laboratório de Entomologia/Patologia de Insetos, da Embrapa-Centro Nacional de Pesquisa de Algodão, em condições controladas de temperatura $\left(25 \pm 1^{\circ} \mathrm{C}\right)$ e umidade relativa $(70 \pm 10 \%)$.

Os insetos foram obtidos no campo, de botões florais de plantas de algodão, pertencentes à cultivar CNPA 7H, e colocados em gaiolas, onde permaneceram até a emergência dos adultos. Quando recém emergidos, 520 adultos do bicudo foram selecionados e agrupados em número de dez indivíduos por recipiente de plástico contendo botões florais sadios, os quais foram mantidos em estufa incubadora B.O.D. até o início do bioensaio.

Isolados do fungo Beauveria bassiana, provenientes de micotecas de diversas instituições (Tabela 1), foram multiplicados em placas de Petri contendo meio de cultura BDA (Batata-Dextrose-Ágar), e incubados, por 15 dias. Foram testados doze isolados de B. bassiana, que constituíram os tratamentos. A testemunha constou da aplicação de água destilada mais espalhante adesivo (Tween-80). As suspensões fúngicas foram preparadas com água destilada, na concentração de $10^{8}$ conídios $/ \mathrm{mL}$. Cada repetição constou de dez insetos adultos, com idade média de oito dias, os quais foram submersos na suspensão fúngica, du- rante dois ciclos de três segundos cada. Após secagem sobre papel de filtro, os insetos foram transferidos para os recipientes de plástico contendo botões florais, trocados diariamente até o término das observações. Para confirmação da patogenicidade do isolado, os insetos mortos foram imersos em hipoclorito de sódio a 3\%, por três segundos, e depois, em água destilada, e em seguida, mantidos em câmara úmida. O porcentual de mortalidade dos insetos foi avaliado durante os dez primeiros dias após a aplicação dos patógenos.

$\mathrm{O}$ teste de germinação de conídios constou da aplicação de $50 \mu \mathrm{L}$ de uma suspensão com $10^{6}$ conídios $/ \mathrm{mL}$ de cada isolado em placas de Petri contendo meio de cultura BDA. As observações foram realizadas a cada duas horas, sobre um total de 400 conídios (100 por repetição).

Os dados de porcentagem de mortalidade dos insetos e de germinação foram utilizados para o cálculo de $\mathrm{TL}_{50}$ (tempo para morte de $50 \%$ dos adultos do bicudo) e $\mathrm{TG}_{50}$ (tempo de germinação de 50\% dos conídios), respectivamente, pelo método de Probit (Finney, 1964). As médias das porcentagens de mortalidade foram corrigidas pela fórmula de Abbott (1925) e comparadas pelo teste de Duncan a 5\% de probabilidade. Foi realizada análise de correlação das variáveis mortalidade $(\%)$ versus $\mathrm{TL}_{50}$, e mortalidade $(\%)$ versus $\mathrm{TG}_{50}$.

\section{Resultados e Discussão}

Os isolados mais virulentos ao bicudo foram, em ordem decrescente: CG138, CNPA02, CNPA03, CG082, CNPA1 e CG070 (Tabela 2). Essas taxas de insetos adultos mortos por infecção de B. bassiana, em laboratório, confirmam resultados de Coutinho \& Oliveira (1991), que obtiveram mortalidade variando de $50 \%$ a $100 \%$ nas concentrações entre $3,72 \times 10^{6}$ a $3,72 \times 10^{10}$ conídios/mL. Gutierrez (1986) obteve mortalidade superior a $50 \%$ quando utilizou concentrações acima de $10^{7}$ conídios $/ \mathrm{mL}$. Por outro lado, os isolados menos virulentos foram, em ordem decrescente: 645, CG458, CNPA04, CG016 e 760. Desses isolados, o 645, o 760 e o CG458 foram obtidos do bicudo-do-algodoeiro (Tabela 1), o que constitui-se numa indicação de que esses isolados foram obtidos de regiões onde o inseto tem provocado dano econômico, e que tais regiões não têm conseguido controlar a praga. Esse fato confirma a necessidade de explorar a variabilidade natural de isolados de fungos entomopatogênicos oriundos de bicudos e insetos não-alvos infectados em áreas de cultivo de algodão com reduzidos prejuízos, em diferentes regiões geográficas, fato, aliás, já exem- 
plificado por Hajek et al. (1990) com o patógeno Entomophaga maimaiga. Esse fungo, originalmente isolado no Japão, do hospedeiro Lymantria dispar, e introduzido nos Estados Unidos por volta de 1910, iniciou epizootia em 1989, e no ano seguinte já atacava larvas de diversos insetos-praga em dez estados norte-americanos. Os valores de $\mathrm{TL}_{50}$ se correlacionaram negativamente, de forma altamente significativa, com a porcentagem de mortalidade $(\mathrm{r}=-0,91)(\mathrm{P}<0,01)$, ou seja, à medida que aumentava a porcentagem de mortalidade dos adultos do bicudo, diminuía o tempo necessário para matar $50 \%$ de sua população (Tabela 2). A patogenicidade diferenciada entre os isolados de $B$. bassiana demonstrou que a espécie do hospedeiro de origem e a região geográfica considerada foram determinantes para obtenção de isolados com ampla variabilidade quanto ao grau de virulência em relação ao bicudo. Por outro lado, o sucesso no emprego de fungos entomopatógenos depende, na maioria das vezes, da correta manipulação dos fatores que interferem na sequiência que as unidades infectivas atravessam desde o momento da dispersão até a infecção do insetoalvo (Sosa-Gomez \& Moscardi, 1992). Dentre esses

Tabela 1. Identificação do isolado, hospedeiro, local de origem e instituição mantenedora do fungo entomopatogênico Beauveria bassiana. Campina Grande, PB, 1999.

\begin{tabular}{llll}
\hline Isolado & Hospedeiro & Local de origem & Instituição \\
\hline CG016 & Diatraea saccharalis (Lepidoptera: Pyralidae) & Londrina, PR & Embrapa-CNPSo \\
CG070 & (Coleoptera: Curculionidae) & Brasília, DF & Embrapa-CENARGEN \\
CG082 & Diabrotica speciosa (Coleoptera: Chysomelidae) & Tucumán, Argentina & ESALQ-USP \\
CG138 & Cosmopolites sordidus (Coleoptera: Curculionidae) & Recife, PE & IPA \\
CG458 & Anthonomus grandis (Coleoptera: Curculionidae) & Londrina, PR & Embrapa-CENARGEN \\
CNPA1 & Polistes sp. (Hymenoptera: Vespidae) & Campina Grande, PB & Embrapa-CNPA \\
CNPA2 & Membracis foliata (Homoptera: Membracidae) & Campina Grande, PB & Embrapa-CNPA \\
CNPA3 & Schistocerca pallens (Orthoptera: Acrididae) & Campina Grande, PB & Embrapa-CNPA \\
CNPA4 & Cycloneda sanguinea (Coleoptera: Curculionidae) & Campina Grande, PB & Embrapa-CNPA \\
IPA147 & Cosmopolites sordidus (Coleoptera: Curculionidae) & Recife, PE & IPA \\
645 & Anthonomus grandis (Coleoptera: Curculionidae) & Recife, PE & UFRPE \\
760 & Anthonomus grandis (Coleoptera: Curculionidae) & Recife, PE & UFRPE \\
\hline
\end{tabular}

Tabela 2. Patogenicidade de isolados de Beauveria bassiana em relação à mortalidade e $\mathrm{TL}_{50}$ (tempo letal de $50 \%$ de insetos) do bicudo-do-algodoeiro e $\mathrm{TG}_{50}$ (tempo para a germinação de 50\% de conídios). Campina Grande, PB, 1999.

\begin{tabular}{|c|c|c|c|c|c|c|c|}
\hline \multirow[t]{2}{*}{ Isolado } & \multirow{2}{*}{$\begin{array}{c}\text { Mortalidade }^{(1)} \\
(\%)\end{array}$} & \multirow{2}{*}{$\begin{array}{l}\mathrm{TL}_{50} \\
\text { (Dias) }\end{array}$} & \multirow[t]{2}{*}{ Equação da reta da $\mathrm{TL}_{50}$} & \multicolumn{2}{|l|}{$\chi^{2}$} & \multirow{2}{*}{$\begin{array}{l}\text { Intervalo de } \\
\text { confiança }^{(2)}\end{array}$} & \multirow{2}{*}{$\begin{array}{c}\mathrm{TG}_{50} \\
\text { (h:min) }\end{array}$} \\
\hline & & & & Calculado & Tabela & & \\
\hline CG 138 & $83 a$ & 5,30 & $Y=2,64572+3,25038 \log x$ & 4,64 & 14,07 & $(4,80 ; 5,85)$ & 18:03 \\
\hline CNPA02 & $65 \mathrm{ab}$ & 6,99 & $Y=3,16660+2,17054 \log x$ & 1,52 & 14,07 & $(6,40 ; 7,64)$ & $19: 02$ \\
\hline CNPA03 & $65 \mathrm{ab}$ & 7,44 & $Y=2,87339+2,44027 \log x$ & 1,58 & 12,59 & $(6,76 ; 8,18)$ & $22: 20$ \\
\hline CG 082 & $60 \mathrm{abc}$ & 8,90 & $Y=2,85391+2,26055 \log x$ & 3,42 & 14,07 & $(7,57 ; 10,46)$ & $19: 35$ \\
\hline CNPA1 & $60 \mathrm{abc}$ & 7,85 & $Y=3,08899+2,13494 \log x$ & 1,85 & 14,07 & $(7,04 ; 8,77)$ & $25: 26$ \\
\hline CG 070 & $55 \mathrm{abc}$ & 8,80 & $Y=2,34543+2,81019 \log x$ & 1,78 & 14,07 & $(8,01 ; 9,67)$ & $20: 26$ \\
\hline IPA147 & $53 \mathrm{bc}$ & 8,02 & $Y=3,44908+1,71522 \log x$ & 3,55 & 14,07 & $(6,62 ; 9,72)$ & $20: 31$ \\
\hline 645 & $50 \mathrm{bcd}$ & 11,06 & $Y=2,97850+1,93666 \log x$ & 2,67 & 14,07 & $(8,94 ; 13,68)$ & $23: 33$ \\
\hline CG458 & $33 \mathrm{bcd}$ & - & - & - & - & - & 17:09 \\
\hline CNPA04 & $30 \mathrm{~cd}$ & - & - & - & - & - & 22:02 \\
\hline CG 016 & $28 \mathrm{~cd}$ & - & - & - & - & - & 19:07 \\
\hline 760 & $15 \mathrm{~d}$ & - & - & - & - & - & $19: 58$ \\
\hline
\end{tabular}

(1) Médias seguidas pela mesma letra não diferem entre si pelo teste de Duncan a 5\% de probabilidade; valores médios de quatro repetições com dez adultos por repetição. ${ }^{(2)}$ Calculado pelo método de Probit. 
fatores destaca-se o efeito deletério da radiação ultravioleta, emanada pelo sol (Ignoffo, 1992). Portanto, a capacidade dos fungos entomopatógenos em tolerar os efeitos deletérios da radiação ultravioleta é de fundamental importância na seleção de isolados.

Neste trabalho, os isolados mais virulentos ao bicudo foram provenientes das regiões Nordeste e Centro-Oeste do Brasil, de maior intensidade de insolação; estes isolados deveriam ser testados em futuros programas de melhoramento genético de fungos entomopatógenos que visem identificar isolados de microorganismos tolerantes à radiação ultravioleta.

Nos isolados estudados, o início da mortalidade dos insetos ocorreu no $2^{\mathrm{o}}$ dia após a inoculação dos patógenos. Tal resultado não coincide com o obtido por Coutinho \& Cavalcanti (1988), os quais constataram que o período de mortalidade do bicudo ocorreu a partir do $4^{\circ}$ dia da inoculação. Essa variação pode ser atribuída às diferenças entre os isolados utilizados, bem como ao método de exposição das suspensões fúngicas em cada experimento, fato já mencionado por Wright \& Chandler (1991). No 6o dia após a inoculação, verificaram-se variações na porcentagem de mortalidade de $5-50 \%$, enquanto as maiores porcentagens de mortalidade variaram de $15-83 \%$ no 10 o dia.

A porcentagem de germinação dos conídios $\left(\mathrm{TG}_{50}\right)$ mostrou variabilidade entre os isolados testados, e não se correlacionou com a patogenicidade $(\mathrm{r}=0,01)(\mathrm{P}>0,05)$; embora a patogenicidade do isolado CG458 tenha sido uma das menores, seu tempo de germinação foi o menor entre os isolados testados (Tabela 2). Contrariamente, o isolado mais patogênico (CG138) apresentou tempo de germinação maior em relação ao isolado CG458. A ausência de correlação entre a patogenicidade e o tempo de germinação do fungo $B$. bassiana tem sido observada por outros pesquisadores (Tigano-Milani et al., 1995; Lecuona et al., 1996), o que indica a importância relativa desse parâmetro biológico nesta espécie de fungo.

O desenvolvimento externo do fungo no inseto adulto coincidiu com observações efetuadas por Coutinho \& Cavalcanti (1988) e Wright \& Chandler (1991): inicia-se entre as articulações do fêmur e da tíbia, membrana cervical e suturas cranianas, recobrindo posteriormente todo o corpo do hospedeiro (Figura 1). As porcentagens de exteriorização

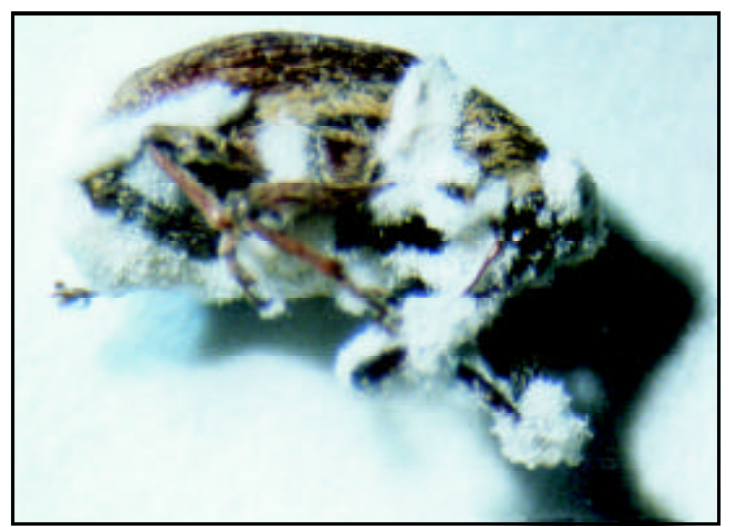

Figura 1. Adulto do bicudo-do-algodoeiro infectado pelo isolado CG138 do fungo Beauveria bassiana.

da doença variaram entre 65-100\% dos exemplares. Nenhum dos insetos mortos na testemunha apresentou estruturas do fungo.

\section{Conclusões}

1. Os isolados de Beauveria bassiana de diferentes hospedeiros e regiões geográficas são diferentes quanto à virulência ao bicudo-do-algodoeiro, Anthonomus grandis.

2. O isolado CG138 de B. bassiana destaca-se como um dos mais virulentos ao bicudo-do-algodoeiro.

\section{Referências}

ABBOTT, W. S. A method of computing the effectiveness of an insecticide. Journal of Economic Entomology, Lanham, v. 18, p. 265-267, 1925.

COUTINHO, J. L. B.; CAVALCANTI, V. A. L. B. Utilização do fungo Beauveria bassiana, no controle biológico do bicudo-do-algodoeiro em Pernambuco. Pesquisa Agropecuária Brasileira, Brasília, v. 23, n. 5, p. 455-461, maio 1988 .

COUTINHO, J. L. B.; OLIVEIRA, J. V. de. Patogenicidade do isolado I-149Bb de Beauveria bassiana (Bals.) Vuil. a adultos de Anthonomus grandis (Coleoptera: Curculionidae). Anais da Sociedade Entomológica do Brasil, Londrina, v. 20, n. 1, p. 199-207, 1991. 
FINNEY, D. J. Probit analysis: a statistical treatment of the sigmoid response curve. Cambridge (Inglaterra) : University Press, 1964. 318 p.

FRANK, W. A.; SLOSSER, J. E. Efficacy of Beauveria bassiana (Balsamo) Vuillemin to control over wintering boll weevils, Anthonomus grandis Boheman. Southwestern Entomologist, Dallas, v. 15, n. 1, p. 77-78, 1990.

GUTIERREZ, G. S. Bioecologia de Anthonomus grandis Boheman, 1843 (Coleoptera: Curculionidae) e seu controle com Beauveria bassiana (Bals.) Vuill. Piracicaba : ESALQ, 1986. 107 p. Dissertação de Mestrado.

HAJEK, A. E.; ELKINTON, J. S.; MAY, B.; WALSH, S. R. A.; SILVER, J. C. Allozyme and restriction fragment length polymorphism analyses confirm Entomophaga maimaiga responsible for 1989 epizootics in North American gypsy moth populations. National Academy of Sciences of the United States of America Proceedings, Washington, v. 87, p. 6979-6982, 1990.

HAJEK, A. E.; LEGER, R. J. S. Interactions between fungal pathogens and insect hosts. Annual Review of Entomology, Palo Alto, v. 39, p. 293-322, 1994.

IGNOFFO, C. M. Environmental factors affecting persistence of entomopathogens. Florida Entomologist, Homestead, v. 75, n. 4, p. 516-525, 1992.

LECUONA, R. E.; MILANI, M. S.; DIAS, B. M. Characterization and pathogenicity of Beauveria bassiana against Diatraea saccharalis (F.) (Lepidoptera: Pyralidae) in Argentina. Anais da Sociedade Entomológica do Brasil, Londrina, v. 25, n. 2, p. 299-307, 1996.
McCOY, C. W.; TIGANO-MILANI, M. S. Use of entomopathogenic fungi in biological control: a world view. Pesquisa Agropecuária Brasileira, Brasília, v. 27, p. 87-93, abr. 1992. Edição especial.

MELO, I. S. de; AZEVEDO, J. L. de. Controle biológico. Jaguariúna : Embrapa-CNPMA, 1998. 264 p.

SOSA-GOMEZ, D. R.; MOSCARDI, F. Epizootiologia: chave dos problemas para o controle de fungos. In: SIMPÓSIO DE CONTROLE BIOLÓGICO, 3., 1992 , Águas de Lindóia. Anais... Jaguariúna : Embrapa-CNPDA, 1992. p. 64-69.

TIGANO-MILANI, M. S.; FARIA, M. R. de; LECUONA, R. E.; SARTORI, M. R.; ARIMA, E. Y.; DIAZ, B. M. Análise de patogenicidade e germinação do fungo Nomuraea rileyi (Farlow) Samson isolado no Distrito Federal. Anais da Sociedade Entomológica do Brasil, Londrina, v. 24, n. 1 , p. 53-60, 1995.

WRIGHT, J. E. Control of the boll weevil (Coleoptera: Curculionidae) with Naturalis-L: a mycoinsecticide. Journal of Economic Entomology, Lanham, v. 86, n. 5, p. 1355-1358, 1993.

WRIGHT, J. E.; CHANDLER, L. D. Development of biorational mycoinseticide: Beauveria bassiana conidial formulation and its application against boll weevil populations (Coleoptera: Curculionidae). Journal of Economic Entomology, Lanham, v. 85, n. 4, p. 1130-1135, 1992.

WRIGHT, J. E.; CHANDLER, L. D. Laboratory evaluation of the entomopathogenic fungus, Beauveria bassiana against the boll weevil (Curculionidae: Coleoptera). Journal of Invertebrate Pathology, San Diego, v. 58, n. 3, p. 448-449, 1991. 